\title{
Turbulence During the Generation of Internal Tide on a Critical Slope
}

\author{
Bishakhdatta Gayen and Sutanu Sarkar* \\ Mechanical and Aerospace Engineering Department, University of California, San Diego, La Jolla, California 92093, USA
}

(Received 15 February 2010; published 27 May 2010; corrected 16 June 2010)

\begin{abstract}
Three-dimensional direct numerical simulations are performed to examine nonlinear processes during the generation of internal tides on a model continental slope. An intense boundary flow is generated in the critical case where the slope angle is equal to the natural internal wave propagation angle. Wave steepening, that drives spanwise wave breaking via convective instability, occurs. Turbulence is present along the entire extent of the near-critical region of the slope. The turbulence is found to have a strong effect on the internal wave beam by distorting its near-slope structure. A complicated wave field with a broadband frequency spectrum is found. This work explains the formation of boundary turbulence during the generation of internal tides in the regime of low excursion numbers.
\end{abstract}

DOI: 10.1103/PhysRevLett.104.218502

PACS numbers: 92.10.Lq, 47.27.E-

Introduction.-Energetic internal waves, commonly known as internal tides, are generated in the ocean by tidal flow interactions with bottom topography: seamounts, ridges, slopes, canyons, etc. [1]. Internal tides are thought to be essential to ocean mixing by providing a dynamical energy pathway from the barotropic tide to propagating internal waves that then lead to mixing in the ocean interior. Observations find enhanced mixing near seamounts [2], ridges [3,4], and continental slopes [5-8]. The reflection of internal waves at a topographic slope with critical angle can lead to turbulent mixing and has been studied in the laboratory and by numerical simulations, e.g., [9]. Here, we investigate the different problem of internal tide generation by barotropic tidal flow over topography.

Theoretical investigations, e.g., [10-12] of internal tide generation rely on linear theory. As discussed by [1], two nondimensional parameters are especially important: (i) the criticality parameter, $\epsilon=\tan (\beta) / \tan \theta$, which is the ratio of the topographic slope $\tan (\beta)$ to the slope of internal wave characteristic $\tan \theta=\sqrt{\left(\Omega^{2}-f^{2}\right) /\left(N_{\infty}^{2}-\Omega^{2}\right)}$ with $\Omega$ the frequency of the barotropic tide, $f$ the Coriolis frequency and $N_{\infty}$ the background value of buoyancy frequency, and (ii) the excursion number, $\mathrm{Ex}=U_{0} / \Omega l$, which is the ratio of excursion of a fluid particle by a tidal velocity, $U_{0}$, to the horizontal length of topography, $l$. Ocean slopes are long with $\epsilon \mathrm{Ex} \ll 1$ so that linear theory applies except for critical slope $\epsilon \simeq 1$. The consequences of nonlinearity in the critical case and with $\epsilon \mathrm{Ex} \ll 1$ is not well understood. Nonlinear aspects have been studied in previous laboratory [13-15] and numerical investigations $[16,17]$. In particular, the authors of [14] find that the resonant wave-slope interaction in the critical case leads to a laminar oscillating boundary layer with maximal velocity. However, turbulence has been overlooked in previous investigations because laboratory experiments were at low Reynolds number and computations were designed for larger scale dynamics. This motivates our three- dimensional direct numerical simulations that resolve turbulence and fine-scale internal waves.

Problem setup.-An oscillatory pressure gradient, $\mathcal{F}=$ $-U_{0} \Omega \cos (\Omega t)$ forces tidal flow over a slope and leads to a barotropic velocity, $U(x) \sin \phi$, where $\phi$ is the tidal phase. As shown in Fig. 1(a), the bottom topography is irregular: a slope between $x=4.5$ and $x=8.0 \mathrm{~m}$ is smoothly connected to horizontal sections before and after it. For simplicity, we do not include rotation.

For all cases, $N_{\infty}=8 \mathrm{rads} / \mathrm{s}$ and $\Omega=1 \mathrm{rad} / \mathrm{s}$, which gives the wave angle $\theta \approx 7^{\circ}$. The kinematic viscosity, $\nu=$ $10^{-6} \mathrm{~m}^{2} / \mathrm{s}$, is that of water. The Prandtl number is chosen to be $\operatorname{Pr}=1$, smaller than the value of $\operatorname{Pr}=5$ for thermal transport in water, so as to avoid an unnecessary increase in computational grid points. Simulations at subcritical slopes $\beta<7^{\circ}$ did not result in turbulence for the range, $\mathrm{Ex}<0.1$, studied here. The Reynolds number is a key parameter and is measured by $\operatorname{Re}=a U_{0} / \nu$ with $a=U_{0} / \Omega$, or by $\operatorname{Re}_{s}=$ $U_{0} \delta_{s} / \nu=\sqrt{2 \mathrm{Re}}$, based on the Stokes boundary-layer thickness, $\delta_{S}=\sqrt{2 \nu / \Omega}$. The salient features of generation at a critical slope will be brought out by discussion of the following case: slope length, $l=3.5 \mathrm{~m}$; current velocity, $U_{0}=0.125 \mathrm{~m} / \mathrm{s} ; \mathrm{Ex}=0.036 ; \epsilon=1 ; \operatorname{Re}=15625 ;$ and $\operatorname{Re}_{s}=177$.

We now place the numerical experiment in context by comparing nondimensional parameters with those corresponding to an oceanic slope. An example of conditions at a continental slope in deep water is as follows: a tidal amplitude of $U_{0}=0.025 \mathrm{~m} / \mathrm{s}$, a tidal frequency of $\Omega=$ $1.4 \times 10^{-4} \mathrm{rad} / \mathrm{s}$ corresponding to the $M_{2}$ tidal period of $12.4 \mathrm{hrs}$, a low latitude with $f=3.5 \times 10^{-5} \mathrm{rad} / \mathrm{s}$ and $N=1 \mathrm{cph}=1.74 \times 10^{-3} \mathrm{rad} / \mathrm{s}$. A representative slope length of $5 \mathrm{~km}$ leads to an excursion number of 0.036 that matches our choice of $\mathrm{Ex}=0.036$. Our critical slope angle $\beta=7^{\circ}$ is slightly larger than the value of $\beta=4.5^{\circ}$ in the ocean example. The Stokes Reynolds number, $\operatorname{Re}_{s}=$ 177 , of our case is smaller than the value of $\mathrm{Re}_{s}=2975$ in 

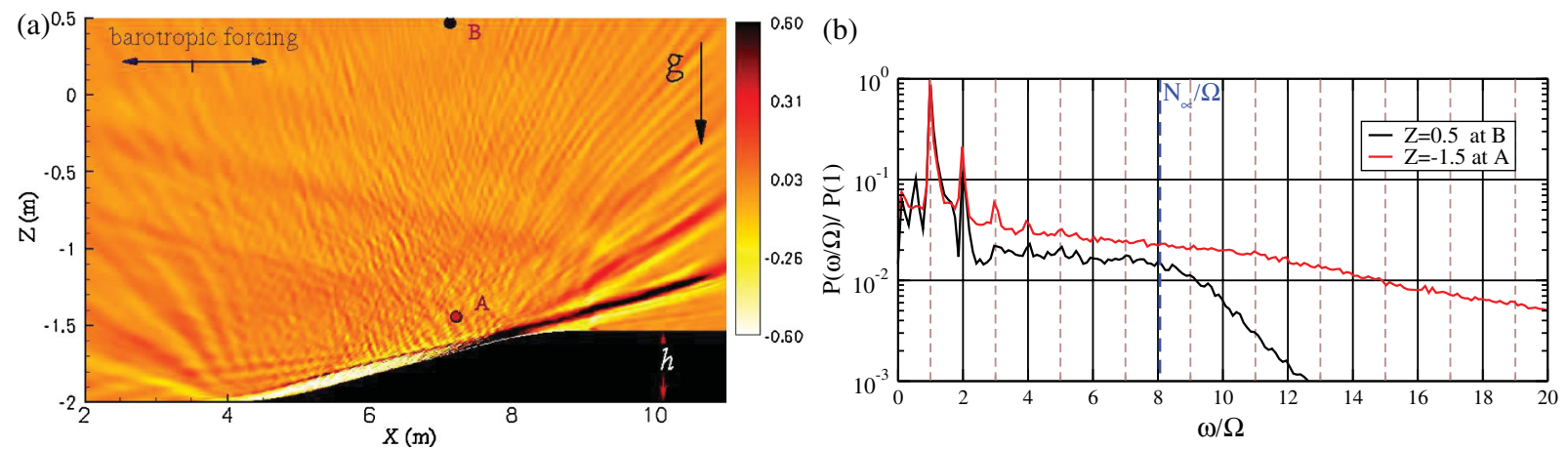

FIG. 1 (color). (a) Internal wave field visualized by a slice of $d w / d z$ field in $x-z$ plane. (b) Power spectra of the baroclinic velocity $u_{\text {bar }}(x, y, z, t)$ field (semi log-scale) at the two locations $A$ and $B$, marked in part (a). The time series is taken over 15 cycles.

the oceanic example but still sufficiently large to exhibit turbulence in the case of critical slope as will be demonstrated.

The three-dimensional, unsteady Navier-Stokes (NS) equations under the Boussinesq approximation for density variation are employed. The NS equations, written in generalized coordinates, are numerically solved on a nonstaggered grid that conforms to the bottom boundary. Spanwise derivatives are treated with a pseudospectral method, and the other spatial derivatives are computed with second-order finite differences. A third-order Runge-Kutta method is used for time-stepping, and viscous terms are treated implicitly with the ADI method. The numerical domain consists of a rectangular box of $13 \mathrm{~m}$ length, $4 \mathrm{~m}$ height, and $1 \mathrm{~m}$ width whose bottom boundary is coincident with the slope topography. The grid size is $260 \times 260 \times 64$ in the $x, z$, and $y$ directions, respectively, with stretching in the $x$ and $z$ directions. The grid spacing is chosen to resolve the viscous turbulence scales and the flow resolution is confirmed by examining the spanwise spectrum. Each simulation is computationally intensive because of the small time step that is required and the large number of cycles that are simulated. Periodicity is imposed in the spanwise, $y$, direction. Zero velocity and a zero value for wall-normal density flux are imposed at the bottom. The upper boundary and the two $x=$ constant boundaries
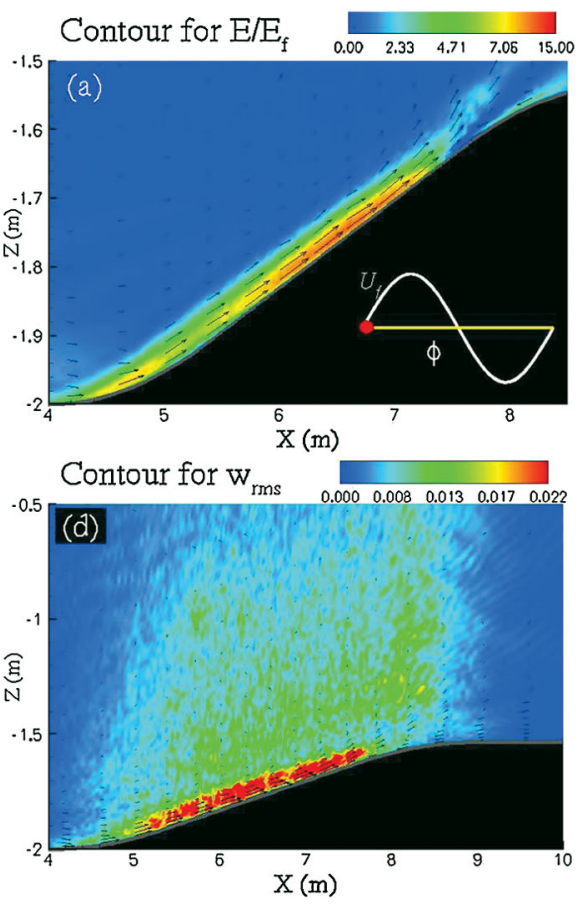
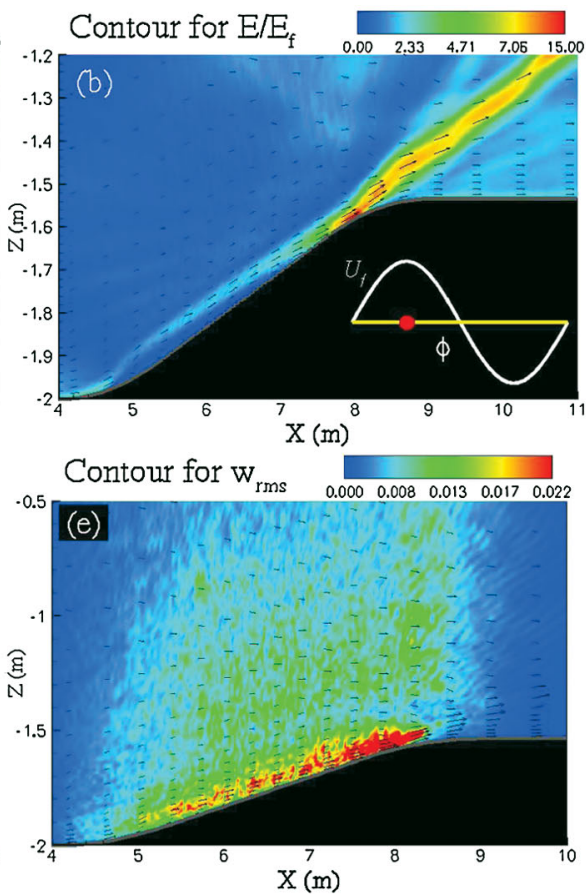
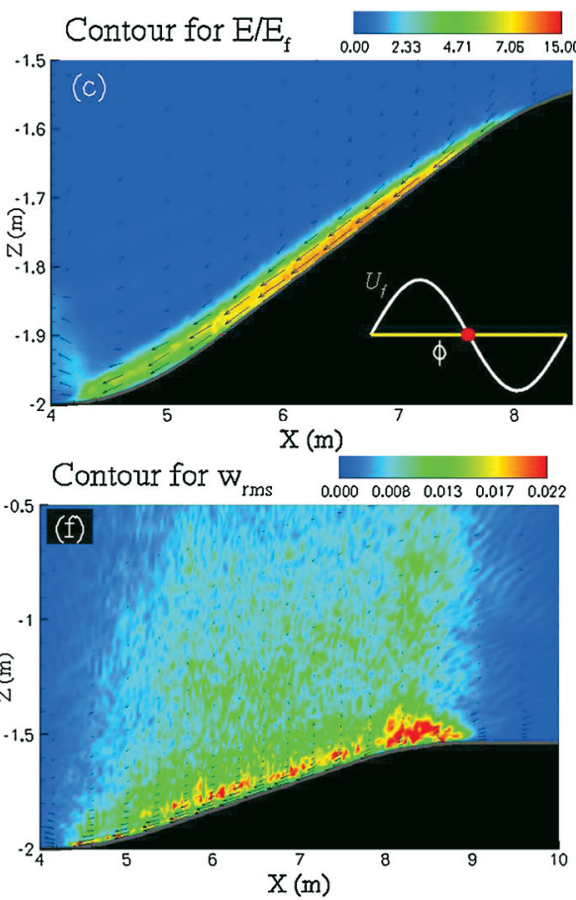

FIG. 2 (color). The resonant generation of the internal tide is shown by the normalized kinetic energy of the flow field $|\langle\mathbf{u}(\mathbf{x}, \mathbf{z}, \mathbf{t})\rangle|^{2} / U_{0}^{2}$ along with the slope topography in black color at time $t=8 T, 8 T+1 / 4 T$ and $8 T+1 / 2 T$ in (a), (b), and (c) respectively. The arrows indicate the instantaneous velocity field. Bottom inset in each top panel figures indicates the corresponding tidal phase. In (d)-(f), vertical turbulence intensity is shown at a time corresponding to that of the figure just above it. 


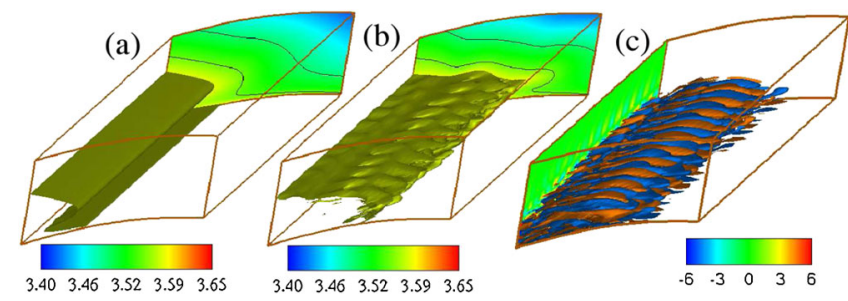

FIG. 3 (color online). Visualization of spanwise instability. (a) Isosurface of density preceding wave breaking, (b) Isosurface of density at wave breaking $(t \simeq T+1 / 4 T)$, (c) streamwise vorticity at same time as part (b).

are artificial boundaries where Rayleigh damping or a "sponge" layer is used.

Results.-The remarkably complex wave pattern, shown in Fig. 1(a), includes energetic internal wave beams coincident with the slope angle, beams at steeper angles, and internal waves generated by boundary-layer turbulence that have a wide range of phase lines. The internal wave field is assessed by a power spectrum analysis of the baroclinic velocity field given by $\mathbf{u}_{\text {bar }}(x, y, z, t)=$ $\mathbf{u}(x, y, z, t)-\mathbf{u}_{\text {baro }}(x, z, t)$, where the barotropic component is the free-stream value of the velocity. Figure 1(b) shows the power spectra at two locations $A$ and $B$ at different heights. The spectra show several temporal harmonics $(n \Omega, n \in \mathbb{N})$, subharmonics $\omega \in[0, \Omega)$ and interharmonics $\left(\omega_{\alpha}+n \Omega, \omega_{\alpha} \in[0, \Omega)\right)$ having significant energy. The discrete spectral peak at the barotropic tidal frequency, $\Omega$, in Fig. 1(b), corresponds to an energetic linear response which, in physical space, corresponds to the strong beams (upward propagation in black and downward in white) shown in Fig. 1(a). The spectrum at point $A$ shows discrete peaks at the second and third harmonics as well as a significant band of waves with $\omega>N$. These super- $N$ waves are generated by high-frequency turbulence inside the boundary layer as in the studies of $[18,19]$. The spectrum at point $B$, further away from the slope, has the same energy at the fundamental and second harmonic observed at point $A$, as well as an additional discrete peak at the first subharmonic. The range of super- $N$ waves in the spectrum is much smaller at point $B$ relative to that at
$A$, since the background does not support freely propagating waves with $\omega>N$.

Resonant wave-slope interactions lead to kinetic energy as large as 15 times that of the barotropic current as shown in Figs. 2(a)-2(c). The intense boundary-layer flow becomes turbulent via the cumulative effect of convective and shear instabilities, as will be shown later. The turbulence interacts with the internal wave beams so that they buckle and twist as shown in Fig. 2(b), a phenomenon not observed in recent experimental studies $[13,14]$ at low Reynolds number. The rms turbulent velocity can be quite large, as much as $50 \%$ of the external barotropic tidal velocity. The spatial distribution of turbulence is illustrated by the vertical component, $w_{\text {rms }}$, in Figs. 2(d)-2(f). Turbulence is convected by the beam to the top of the slope in Fig. 2(e). The patches of turbulence at the top corner of the slope in Fig. 2(f) correspond to residual turbulence generated during the previous phase. There is a significant amount of turbulence observed even outside the boundary layer.

We have identified two mechanisms, convective and shear instability, that cause transition to turbulence. The intensified velocity during the upslope motion becomes large enough to steepen the wave near the bottom boundary. Eventually, a spanwise instability develops as illustrated by Fig. 3. The density isosurface which is smooth in Fig. 3(a) develops spanwise corrugations during the instability as shown in Fig. 3(b). These coherent corrugations are associated with pairs of counter-rotating streamwise vortices as shown in Fig. 3(c). The wavelength of the spanwise instability is intrinsic to the flow and is not observed to change upon changing the spanwise domain length. Spanwise instability has been implicated in other examples of breaking [20,21] of free internal waves. Terms calculated to understand the energetics of the observed instability are show in Fig. 4 along with isopycnals. The overturned isopycnals show the kinematically unstable structure of the wave due to wave steepening. The positive buoyancy flux in the overturned region is significant as illustrated by Fig. 4(b). The buoyancy flux transfers energy from the potential mode to the kinetic mode resulting in the corresponding patch of turbulent kinetic energy (TKE)
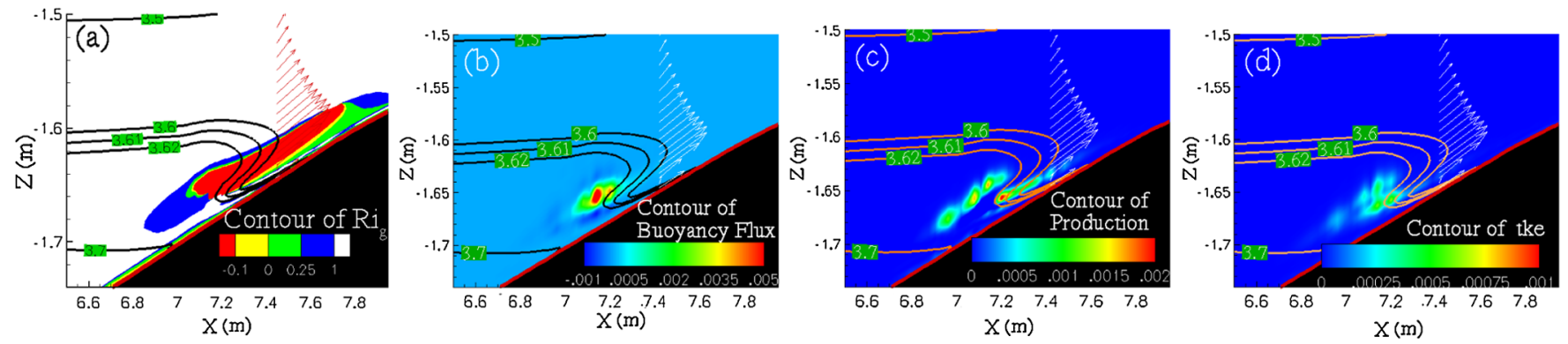

FIG. 4 (color online). Contour of $\mathrm{Ri}_{g}$, buoyancy flux, production and turbulent kinetic energy at $t=t+1 / 4 T$ in (a), (b), (c) and (d), respectively. Arrows indicate the vector field at a certain position at that instant of time. Isopycnals shown to indicate wave breaking via wave steepening. 


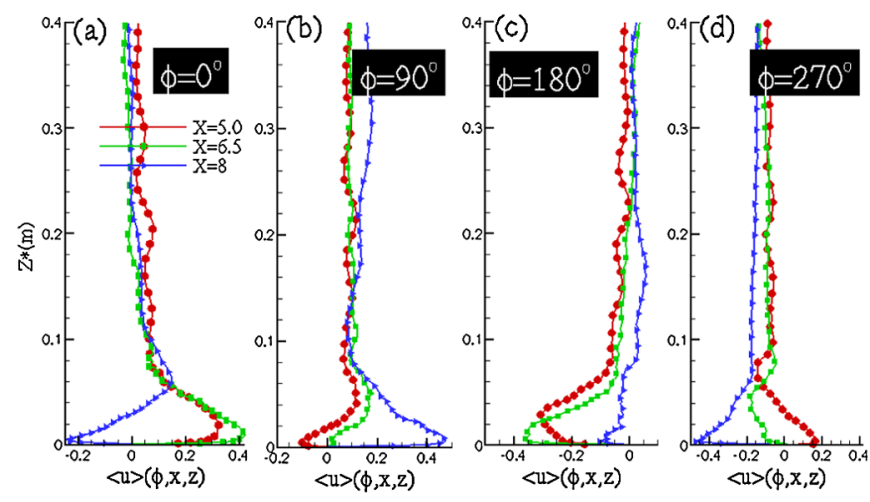

FIG. 5 (color online). Streamwise velocity profile as a function of vertical height and phase at three different locations $X=5 \mathrm{~m}$, 6.5 and $8 \mathrm{~m}$ shown in Fig. 1(a) at $t=8 T, 8 T+1 / 4 T, 8 T+$ $1 / 2 T$ and $8 T+3 / 4 T$ in (a), (b), (c), and (d), respectively.

observed in Fig. 4(d). The diagnostics show that a convective (buoyancy driven) instability is clearly present. Nevertheless, the role of shear instability cannot be overlooked. Figure 4(a) shows the gradient Richardson number $\mathrm{Ri}_{g}=N^{2} / S^{2}$ with $N$ and $S$ the local values of mean buoyancy frequency and mean shear, respectively. A substantial region with low $\mathrm{Ri}_{g}$ can be seen. There is significant shear production by turbulence (although smaller than the peak buoyancy flux at this time) corresponding to that region as shown by Fig. 4(c) as well as corresponding patches of TKE in Fig. 4(d). The coherent counterrotating vortex pairs interact and, after a short time, there is breakdown into fine-scale turbulence. During subsequent cycles, turbulence levels increase and decrease corresponding to changes in the near-bottom velocity.

The internal tide causes the mean velocity to have a complex behavior as can be seen in Fig. 5. Velocity profiles are shown at 3 locations: $x=5 \mathrm{~m}$ at the beginning of the slope, $x=6.5 \mathrm{~m}$ at midslope, and $x=8 \mathrm{~m}$ at the end of the slope. Figure 5 shows that, near the bottom, there is a large increase of horizontal velocity with respect to the external (barotropic) current and, furthermore, this velocity shows significant variation among the 3 locations. At the midslope location, $x=6.5 \mathrm{~m}$, the near-bottom velocity reaches its maximum value at $\phi=0^{\circ}$ and $\phi=180^{\circ}$ when the barotropic tide is minimum and, consequently, the turbulence level on most of the slope is maximum when the barotropic current is minimum.

Conclusions.-Oscillating flow over a long slope (low excursion number) is examined using three-dimensional numerical simulations. In the critical case, the generated internal wave propagates at an angle identical to the slope angle. We show numerically that, owing to the resonant wave-slope interaction in the critical case, the boundary flow is strongly intensified as in the laboratory experiment of [14] and, furthermore, at the higher Reynolds number of the numerical simulations relative to the laboratory cases, the flow becomes turbulent. Transition to turbulence involves wave steepening at the slope that is followed by a convective (buoyancy driven) instability that leads to threedimensional fluctuations in the form of coherent streamwise vortices. In addition, there is shear instability associated with subcritical Richardson numbers in the intensified boundary flow. Recent observations [6-8] find hot spots of turbulence in the vicinity of near-critical slopes, and critical reflection of incident internal waves has been suggested in the literature as a possible explanation. Our results imply that the mechanism of critical generation must also be considered as a potential explanation for enhanced turbulence near slopes.

We are grateful for support provided through NSF Grant No. 0825705.

*Corresponding author. sarkar@ucsd.edu

[1] C. Garrett and E. Kunze, Annu. Rev. Fluid Mech. 39, 57 (2007).

[2] R. G. Lueck and T. D. Mudge, Science 276, 1831 (1997).

[3] D. L. Rudnick et al., Science 301, 355 (2003).

[4] J. M. Klymak, J. N. Moum, J.D. Nash, E. Kunze, J. B. Girton, G. S. Carter, C. M. Lee, T. B. Sanford, and M. C. Gregg, J. Phys. Oceanogr. 36, 1148 (2006).

[5] D. A. Cacchione, L. F. Pratson, and A. S. Ogston, Science 296, 724 (2002).

[6] J.N. Moum, D.R. Caldwell, J.D. Nash, and G. D. Gunderson, J. Phys. Oceanogr. 32, 2113 (2002).

[7] J. D. Nash, E. Kunze, J. M. Toole, and R. W. Schmitt, J. Phys. Oceanogr. 34, 1117 (2004).

[8] J.D. Nash, M.H. Alford, E. Kunze, K. Martini, and S. Kelly, Geophys. Res. Lett. 34, L01605 (2007).

[9] D. N. Slinn and J. J. Riley, Theor. Comput. Fluid Dyn. 11, 281 (1998).

[10] T. H. Bell, J. Geophys. Res. 80, 320 (1975).

[11] P. G. Baines, Deep-Sea Res. Part A 29, 307 (1982).

[12] S. G. L. Smith and W. R. Young, J. Fluid Mech. 495, 175 (2003).

[13] L. Gostiaux and T. Dauxois, Phys. Fluids 19, 028102 (2007).

[14] H. P. Zhang, B. King, and H. L. Swinney, Phys. Rev. Lett. 100, 244504 (2008).

[15] P. Echeverri, M. R. Flynn, K. B. Winters, and T. Peacock, J. Fluid Mech. 636, 91 (2009).

[16] S. Legg, J. Phys. Oceanogr. 34, 156 (2004).

[17] A. S. Korobov and K. G. Lamb, J. Fluid Mech. 611, 61 (2008).

[18] J. R. Taylor and S. Sarkar, J. Fluid Mech. 590, 331 (2007).

[19] B. Gayen, S. Sarkar, and J. R. Taylor, J. Fluid Mech. 643, 233 (2010).

[20] K. B. Winters and E. A. D'asaro, J. Fluid Mech. 272, 255 (1994).

[21] C. R. Koudella and C. Staquet, J. Fluid Mech. 548, 165 (2006). 